\title{
Effect of thermal dispersion on transient natural convection in a wavy-walled porous cavity filled with a nanofluid: Tiwari and Das' nanofluid model
}

\begin{abstract}
Transient natural convection in a porous wavy-walled cavity filled with a nanofluid has been studied numerically. The domain of interest is bounded by vertical flat and horizontal wavy walls having constant temperatures. The unsteady governing equations formulated in dimensionless stream function and temperature, within the Darcy-Boussinesq approximation and the mathematical nanofluid model proposed by Tiwari and Das in the presence of thermal dispersion with corresponding initial and boundary conditions have been solved numerically using an iterative implicit finite-difference method. The main objective is to investigate the effect of the dimensionless time, thermal dispersion parameter and solid volume fraction parameter of nanoparticles on the fluid flow and heat transfer characteristics. Results are presented in the form of streamlines, isotherms and distributions of the average Nusselt number at the bottom wavy wall.
\end{abstract}

Keyword: Porous cavity; Wavy wall; Thermal dispersion; Nanofluid; Numerical results 
